\title{
Les Séminaires des Missions-Étrangères de Paris et de Québec
}

\section{Honorius Provost}

Volume 38, 1971

La genèse de la Société des Missions-Étrangères de la province de Québec

URI : https://id.erudit.org/iderudit/1007262ar

DOI : https://doi.org/10.7202/1007262ar

Aller au sommaire du numéro

Éditeur(s)

Les Éditions Historia Ecclesiæ Catholicæ Canadensis Inc.

ISSN

0318-6172 (imprimé)

1927-7067 (numérique)

Découvrir la revue

Citer cet article

Provost, H. (1971). Les Séminaires des Missions-Étrangères de Paris et de Québec. Sessions d'étude - Société canadienne d'histoire de l'Église catholique, 38, 1-16. https://doi.org/10.7202/1007262ar

Tous droits réservés @ Les Éditions Historia Ecclesiæ Catholicæ Canadensis Inc., 1972
Ce document est protégé par la loi sur le droit d'auteur. L'utilisation des services d'Érudit (y compris la reproduction) est assujettie à sa politique d'utilisation que vous pouvez consulter en ligne.

https://apropos.erudit.org/fr/usagers/politique-dutilisation/ 


\section{Les Séminaires des Missions-Étrangères de Paris et de Québec}

Il n'y a pas de génération spontanée en ce bas monde, pas plus dans le domaine des institutions, le domaine spirituel, que dans le domaine biologique. L'histoire de nos fondations est même très souvent celle d'une longue et pénible gestation. Pareillement, leur succès ou leur pérennité s'apparentent parfois étrangement à la somme de leurs épreuves, considérées au moins du point de vue de la foi. L'Église naissante n'avait-elle pas elle-même trouvé son triomphe dans le sang de ses innombrables martyrs?

Il y a à peine trois cents ans - nous sommes au Nouveau-Monde que $\mathrm{M}^{\mathrm{gr}}$ de Laval, de passage à Paris, décrétait la fondation du Séminaire de Québec, le 26 mars 1663. Nous avons conservé précieusement, de cette érection canonique, le beau et vénérable parchemin, corroboré par un document similaire, pour l'érection civile, les lettres patentes de Louis XIV.

Cette fondation avait une portée bien plus vaste que celle qu'on entendrait aujourd'hui, en employant la même formule. Elle établissait le séminaire " pour servir de clergé à cette nouvelle Église »; ce sont les termes mêmes placés en vedette dans l'acte d'érection. Dans cette Nouvelle-France encore en état de mission, $\mathbf{M}^{\text {gr }}$ de Laval voulait un diocèse à forme communautaire, une vie de famille du clergé autour de son évêque et le séminaire serait le siège, le centre, le cœur de la communauté des prêtres séculiers ou diocésains, tous sollicités d'en faire partie, avec une garantie de sécurité pour leur vie spirituelle et temporelle, moyennant l'apport de leur dévouement pastoral et l'abandon de leurs rentes ou revenus casuels, de quelque nature qu'ils fussent. Comme dans le collège apostolique, il ne devait y avoir au séminaire qu'un fonds commun pour l'entretien de tous ses membres, et même de l'évêque, considéré comme le père de la famille.

C'est à cette institution, du reste, que $\mathbf{M}^{\mathrm{gr}}$ de Laval remettait l'intendance temporelle du diocèse, ou plutôt du vicariat apostolique, avec trois fonctions spécifiques: le ministère paroissial, l'apostolat missionnaire et la formation d'un clergé national. C'était une attribution, un mandat, sans être une autonomie; car le séminaire demeurait vraiment épiscopal et diocésain et, comme tel, soumis à l'Ordinaire, selon les canons du Concile de Trente, expressément invoqués dans l'acte d'érection ${ }^{1}$.

1 Auguste-Honoré Gossenr, Vie de Mor de Laval, I, page 387. 
Cependant, tout cela ne s'était pas conçu sans une arrière-pensée et même, pouvons-nous présumer, sans un début de convention avec le Séminaire des Missions Étrangères, alors en voie d'organisation à Paris, sur la rue du Bac. Quand les deux premiers associés, les abbés Michel Gazil et Armand Poitevin, s'y agrégèrent quatre autres compagnons ecclésiastiques, par un acte juridique du 10 mars $1664^{2}$, on put dire que la vie de ce séminaire commençait pour de bon. Celle de Séminaire de Québec avait débuté à l'arrivée de $\mathrm{M}^{\mathrm{gr}}$ de Laval et de ses compagnons à Québec, le 15 septembre précédent.

Au printemps de 1664, les premiers vaisseaux apportèrent de France la nouvelle de l'établissement définitif de Séminaire de Paris. Déjà celui de Québec put se considérer en pratique comme réuni et soumis à l'autre, comme à sa maison mère, bien que les deux institutions fussent légalement distinctes. C'est pourquoi, devançant les formalités requises, on se mit aussitôt à placer en vedette le Séminaire des Missions Étrangères. C'est à lui, bien qu'au profit du Séminaire de Québec, que le père Jérôme Lalemant sacrifie, le 16 août 1664 , un cinquième de la pension royale accordée aux Jésuites; c'est à lui que $\mathrm{M}^{\mathrm{gr}}$ de Laval unit la cure de Québec, le 15 septembre suivant, dans cet acte d'érection pourtant si important au point de vue juridique; et le premier curé, $M$. Henri de Bernières, se voit qualifier, en l'occurence, de prêtre du "Séminaire des Missions Étrangères de Paris », alors qu'il ne l'était pas encore et qu'il ne le serait jamais, à proprement parler. De toute évidence, on avait hâte et on anticipait.

Rien d'étonnant, alors, que $\mathbf{M}^{\mathrm{gr}}$ de Laval mette sur les premiers navires en partance pour outre-mer un double document sur le même thème: une invitation aux Messieurs de Paris à venir s'établir à Québec, datée du 20 août 1664, et, deux jours plus tard, une permission en forme canonique ${ }^{3}$. Dans le préambule de celle-ci, $\mathrm{M}^{\mathrm{gr}}$ de Laval allègue, en effet, " la demande que nous auraient faite Messieurs les Ecclésiastiques du Séminaires des missions étrangères établi à Paris, au faubourg St-Germain, d'agréer l'établissement d'une de leurs maisons dans notre diocèse " ... Si telle demande a été faite, elle est aujourd'hui disparue; ou bien elle n'était que verbale.

Une première observation au texte de ces deux pièces ne laisse pas de nous intriguer. Dans la lettre, $\mathrm{M}^{\mathrm{gr}}$ de Laval promet * un logement préparé et un fond suffisant pour commencer un petit établissement ". Dans la permission officielle, il déclare: "Nous avons annexé et annexons pour le présent et à toujours irrévocablement à la maison du dit corps qui sera établie à Québec, la paroisse du dit Québec, ... avec son

2 Actes primitifs concernant lEtablissement du Séminaire des Missions Etrangères, Paris, 1750, pages 19 et suiv.

3 Archives du Séminaire de Québec, cartable Séminaire 2, $\mathrm{n}^{\circ / 5} 51$ et 28b. 
presbytère, revenus et autres dépendances "... Alors que, nous le savons bien, tous les articles mentionnés ci-dessus étaient déjà l'affaire du Séminaire de Québec, en bonne forme et de notoriété publique, comment se fait-il que le nom de cette institution n'y soit pas prononcé une seule fois ? On ne devait rien ignorer de cela en France. Est-ce une forme de délicatesse de la part de l'évêque envers ses amis de Paris, évitant de leur faire sentir qu'une fondation les a déjà précédés à Québec ? Est-ce une façon d'humilité et de désintéressement de sa part, en ne comptant pour rien le fruit de ses démarches et de ses sacrifices?

Quoi qu'il en soit, les officiers du Séminaire de Paris tiendront un autre langage. Ils dissiperont l'équivoque en remettant le Séminaire de Québec en tête de la manchette. Dans leur convention du 29 janvier $1665^{4}$, où deux d'entre eux, MM. Armand Poitevin et Jacques Bertot, font partie distincte, comme procureurs de $M^{\text {gr }}$ de Laval, il n'est question ni d'érection d'un nouveau séminaire, ni d'absorption d'un séminaire par l'autre, mais d'union et d'annexion de deux séminaires distincts, déjà existants et devant conserver leur individualité. Les directeurs acceptent, nous dit le texte, "l'union et annexe du dit Séminaire de Québec et de la ditte cure et paroisse de Notre Dame du dit lieu à leur dit Séminaire de Paris, ensemble les donations des maisons et presbytère, terres, revenus et droits, et autres dépendances *...

Plus que cela, le Séminaire des Missions Étrangères, pour prévenir tout malentendu, établit deux réserves significatives, deux bornes précises aux frontières de cette union, à savoir, "que chaque maison ou Séminaire sera soutenu et nourri des revenus qui lui seront propres et destinés et portera ses charges, que le dit Séminaire de Paris ne sera point tenu d'envoyer des sujets et ouvriers en Canada qu'autant qu'il s'en présentera de capables et qu'il le pourra ».

Ajoutons immédiatement qu'une frontière s'établit par la suite, dans la pratique, accentuant la distinction entre les deux séminaires: c'est que les prêtres agrégés à l'un ne l'étaient pas à l'autre nécessairement. Il n'y eut que des exceptions, quelques recrues venues de France après leur agrégation à la maison de Paris; on les agrégeait à Québec, dès leur arrivée, mais ils conservaient leur première agrégation et plus d'une fois on les a vus retourner finir leurs jours à leur maison d'origine.

M. Adrien Launay, dans son Histoire générale de la Socièté des Missions Étrangères ${ }^{5}$, signale la même chose, sous un autre angle, en parlant des prêtres destinés au Séminaire de Quebec: «Entre leur situation dans la Société et celle des missionnaires d'Extrême-Orient, il y eut toujours ces deux différences: ils étaient libres d'accepter ou de refuser d'al-

4 Ibidem, $\mathrm{n}^{\circ} 28 \mathrm{a}$.

b Paris, 1894, volume I, page 161. 
ler au Canada et ils ne recevaient pas la patente qui associait directement aux Missions Étrangères; tandis que les missionnaires d'Asie devaient, sous peine d'exclusion, accepter la mission qu'on leur désignait, et étaient incorporés dès leur départ. »

Dans les lettres patentes d'union émises par $\mathrm{M}^{\mathrm{gr}}$ de Laval le 22 août 1664, il était dit: "Pour le regard du Supérieur de la dite maison établie au dit Québec, il sera choisi et nommé par Messieurs du dit Séminaire de Paris suivant leurs règles et constitutions, et prendra ensuite notre bénédiction pour en exercer la charge." La même clause revient identiquement dans le contrat d'union du 29 janvier 1665. En conséquence, deux mois après, le 29 mars, les officiers du Séminaire de Paris, tenant compte à nouveau d'un ordre de choses déjà existant, nommaient l'abbé Henri de Bernières " pour premier supérieur du dit Séminaire établi à Québec pour la conversion des infidèles, pendant le temps de trois années ... tant pour le spirituel que pour le temporel, à l'avantage des ecclésiastiques qui y seront élevés et rendus capables de servir $l^{\prime}$ Église ${ }^{6}$. Q Quant aux "autres officiers subalternes audit supérieur, comme assistant, préfet des choses spirituelles et procureur ", le Séminaire de Paris laissait aux prêtres de Québec le soin de les choisir euxmêmes, par voie de suffrage.

A partir de ce moment, c'est-à-dire deux ans à peine après sa fondation, le Séminaire de Québec perdait donc son autonomie en grande partie, pour devenir une succursale, ou mieux une dépendance du Séminaire des Missions Étrangères. $M^{g r}$ Taschereau conclut là-dessus de la façon suivante ${ }^{7}$ : "Il était à la fois séminaire épiscopal et diocésain et comme tel soumis à l'Évêque, selon les canons du Concile de Trente, et séminaire des Missions Étrangères, dépendant de celui de Paris pour le temporel et pour la nomination de ses supérieurs. 》 Cette dualité de caractère sera une pierre d'achoppement et soulèvera des malentendus dans la suite. Il eût mieux valu préciser dès le début que le * Séminaire des Missions Étrangères établi à Québec " faisait fonction subsidiaire de séminaire diocésain ou le dirigeait tout simplement, en continuant d'être une communauté de prêtres voués avant tout au service du diocèse et des missions, comme l'avait conçue $\mathrm{M}^{\mathrm{gr}}$ de Laval, dès la fondation, en 1663, et comme il la désirait encore, le 22 août 1664, en donnant aux prêtres des Missions Étrangères «tout pouvoir... d'enseigner les peuples pour ce qui regarde la vie et les vertus chrétiennes, par les prédications, catéchisme, conférences, retraites spirituelles, et autres exercices de dévotion, aussi d'aller en mission dans tous les lieux » de son diocèse et de sa juridiction. Dans une permission aussi étendue, il ne mentionnait même pas le but spécifique de la formation du clergé.

6 Archives du Séminaire de Québec, cartable Séminaire 1, $\mathrm{n}^{\circ} 10$.

7 Ibidem, Histoire manuscrite du Séminaire, pages 30 et suiv. 
Or, $\mathrm{M}^{\mathrm{gr}}$ de Laval et ses collaborateurs avaient pensé à cela; ils avaient prévu les inconvénients de la formule. Ce qu'on leur envoyait de Paris n'était pas ce qu'ils avaient demandé, ne leur donnait pas satisfaction en tous points. C'est pourquoi ils continuent à procéder suivant leur plan à eux. Ils transportent au Séminaire de Paris le titre de l'emplacement que $M^{\text {gr }}$ de Laval vient d'acheter de la veuve de Guillaume Couillard pour donner de l'espace vital à son séminaire; ils demandent au Saint-Siège de confirmer par décret l'union du Séminaire et de la cure de Québec au "Séminaire des Missions étrangères de Québec "; enfin, ils sollicitent le Séminaire de Paris d'accorder un nouvel acte séparé, a dans lequel il ne soit fait mention du Séminaire épiscopal ni d'annexer la cure de Québec; mais seulement d'une maison de la congrégation du Séminaire des Missions Étrangères, afin d'éviter les difficultés que nous pourraient causer à la suite les Évêques : ou en retirant leurs séminaristes de notre maison et formant un autre séminaire, ou en nous ôtant la cure, s'ils le pouvaient. Car, en ce cas, nous demeurerions établis et bâtis sur un fond qui nous appartient, indépendamment du Séminaire épiscopal et de la cure et presbytère »...

$\mathrm{M}^{\mathrm{gr}}$ de Laval se mettait donc martel en tête par précaution pour l'avenir, rendu circonspect par les intrigues gallicanes. Terminant, en 1667, la première construction, en bois, de son séminaire, il y apposait déjà au fronton le monogramme S.M.E. (Seminarium Missionum Exterarum). Mais le Séminaire de Paris passa outre à ce désir de fusion pure et simple et s'en tint à sa formule d'union bilatérale du 29 janvier 1665. Refus de Paris, silence de Rome : notre séminaire demeura sur une équivoque en s'appelant: "Séminaire des Missions Étrangères de Québec ", ou, pire encore: "Séminaire des Missions Étrangères étably à Québec ».

Gardant son individualité, il était placé en tutelle; une tutelle qui pourrait paraître encombrante, à certains moments, mais demeurait bienfaisante, tant pour le recrutement des sujets que pour la direction spirituelle et la subsistance matérielle. Si nous ne l'avions pas eue, que serait devenu le Séminaire de Québec ?

L'histoire de cette union n'est pas allée sans incidents, pendant les quelque cent ans qu'elle a prévalu juridiquement. Mais il est intéressant autant qu'édifiant de constater que tous les assauts majeurs qu'elle eut à affronter lui vinrent de l'extérieur et non de quelque friction interne entre les deux parties contactantes. Tout au plus pouvons-nous signaler, au second quart du XVIII' siècle, un certain malaise passager entre la direction trop française et les sujets peut-être trop canadiens de la maison de Québec. Réaction qui n'a rien de nature à scandaliser, étant bien humaine, sinon bien excusable, même entre gens d'Église ! 
Quand $M^{\mathrm{gr}}$ de Laval fut enfin devenu évêque en titre de Québec, le $1^{\text {er }}$ octobre 1674, il crut devoir reprendre un à un les actes juridiques qu'il avait posés, se figurant, à tort ou à raison, qu'il n'avait pu leur conférer de valeur permanente, en tant que simple vicaire apostolique.

Étant donc à Paris, le 19 mai 1675, avec comme secrétaire l'abbé Charles Glandelet, qu'il vient d'embaucher pour son œuvre au Canada, il dicte un nouvel acte d'union des deux séminaires ${ }^{8}$, mais sans les appréhensions qui l'obsédaient dix ans auparavant. Se référant à l'érection primitive du Séminaire de Québec et aux lettres patentes de 1663, et alléguant les précieux services déjà rendus par le Séminaire de Paris, \& nous avons estimé, dit-il, ne pouvoir faire chose plus conforme aux intentions de Sa Majesté, ni plus solidement pourvoir à la conservation du dit Séminaire de Québec dans le même esprit ecclésiastique et des missions, que de lui procurer la continuation du même gouvernement que nous avons déjà éprouvé si utile, en l'unissant et annexant audit Séminaire de $\mathrm{Pa}$ ris ... d'où il a reçu jusqu'à présent son principal secours... À ces causes, ... nous avons uni et annexé, unissons et annexons à perpétuité le dit Séminaire de Québec, ses maisons et bâtiments, jardins, emplacements, seigneuries, terres, possessions, revenus généralement quelconques avec autres dépendances d'icelui présents et à venir, au dit Séminaire établi à Paris pour la conversion des infidèles, sans que le dit Séminaire de Québec ni ceux qui y sont demeurants en puissent distraire, vendre ou aliéner aucune partie ni même les engager sans le consentement et permission des Sieurs Directeurs du dit Séminaire de Paris, qui nommeront et choisiront tel Supérieur que bon leur semblera, pour régir et gouverner selon leur constitution le dit Séminaire de Québec, lequel Supérieur prendra notre bénédiction et confirmation pour exercer sa charge."

Donnant en outre la juridiction ecclésiastique aux prêtres envoyés de Paris au Séminaire de Québec, l'évêque précise: a $\mathrm{A}$ condition toutefois d'être soumis à Nous et à nos successeurs Évêques en toutes les fonctions ecclésiastiques qui regardent l'assistance et l'instruction du prochain, et quant au reste ils dépendront de leur Supérieur et du dit Séminaire de Paris. "

Le jour même de l'émission de l'Acte d'Union, les supérieurs de Paris en signaient l'acceptation. $\mathrm{M}^{\mathrm{gr}}$ de Laval, devant $\mathrm{s}^{\prime}$ embarquer presque aussitôt pour le Canada, leur laissa le soin de poursuivre l'obtention des lettres patentes du roi, pour légaliser civilement l'union conclue, chose laissée en suspens à la première union, en 1665 . On ne réussira à les obtenir qu'au mois d'avril $1676^{\circ}$.

D'autres formalités suivront avant l'enregistrement des lettres patentes au Parlement de Paris, entre autres l'enquête de commodo et incom-

8 Ibidem, cartable Séminaire $1, n^{\circ} 1$.

- Ibidem, cartable Séminaire 11, $\mathbf{n}^{\circ} \mathbf{2}$. 
modo, c'est-à-dire l'audition de témoins désintéressés, ecclésiastiques et laïcs, pour constater publiquement l'utilité de l'union des deux séminaires ${ }^{10}$. C'est pourquoi l'enregistrement ne put avoir lieu que le 10 février 1678. Que de chinoiseries, serions-nous tenté de dire aujourd'hui ! Mais n'oublions pas que les ronds-de-cuir sont une vieille institution; le grand siècle avait les siens, qu'il fallait tenir occupés, sans manquer de les récompenser...

Et, pourtant, le croira-t-on, $\mathrm{M}^{\mathrm{gr}}$ de Laval ne se sentit pas encore pleinement rassuré après toutes ces procédures. Redoutant avec raison les ingérences gallicanes sur les institutions canoniques même les mieux établies, il cherchait un point d'appui sur le roc inébranlable de Pierre. Traduisons du latin un extrait de sa lettre au Pape saint Innocent XI, du 13 novembre $1678^{11}$ :

* Une autre chose qui, je pense, ne déplaira pas à Votre Sainteté; pour que les clercs en train de se former à la discipline ecclésiastique puissent plus facilement et plus commodément être instruits des fonctions pastorales, j'ai pourvu à l'érection de la paroisse de Québec, sous le titre de la Bienheureuse (Vierge) Marie, et je l'ai unie à perpétuité au Séminaire des Nations étrangères fondé à Paris, mais ayant aussi un siège permanent à Québec, auquel Séminaire j'ai pareillement confié le Séminaire épiscopal (...) toutes choses, Très Saint-Père, que je supplie fortement Votre Sainteté de confirmer par décret spécial, pour que cet arrangement, destiné à servir l'Église, demeure solide et reconnu et ne puisse plus jamais être changé, ni par moi, ni par aucun de mes successeurs. D Où l'on voit que $\mathrm{M}^{\mathrm{gr}}$ de Laval se méfiait peut-être autant de lui-même que des autres.

Dans les années qui suivent, le Séminaire de Québec entre en possession de vastes terrains, par donation de $\mathbf{M}^{\mathrm{gr}}$ de Laval et par d'autres acquisitions. Si bien que les directeurs de la maison, se sentant paralysés dans leur administration par les clauses de l'union de 1675, demandent et obtiennent de Paris, le 6 juin $1682^{12}$, un acte de désistement. Les directeurs de Paris les autorisaient à faire des transactions immobilières et à choisir même leur supérieur, à la seule condition de faire approuver ces décisions, pour fin de surveillance. $\mathbf{M}^{\mathrm{gr}}$ de Laval, toujours condescendant, posa son approbation autographe au bas du document, le 4 novembre 1682.

$10 \quad$ Ibidem, $\mathbf{n}^{\circ} 31$.

11 Altera nova positio super virtutibus, Rome, Imprimerie du Vatican, 1956, pages 137 et suiv.

12 Archives du Séminaire de Québec, cartable Séminaire 1, n 24. 
L'année suivante, le 6 mars ${ }^{13}$, Paris approuvait les règlements du Séminaire de Québec, enfin codifiés pour une première fois, après des pourparlers assez laborieux, non seulement en vue d'y entériner les clauses de l'acte de désistement dont il vient d'être question, mais aussi dans le but de faire reconnaître des usages établis à Québec, notablement différents de ceux du Séminaire de Paris, les deux maisons étant ellesmêmes différentes dans leur destination.

Sous la gouverne d'un nouveau supérieur, jeune et dynamique, l'abbé Jacques-Charles de Brisacier, le Séminaire de Paris prenait plus d'intérêt pour celui de Québec. "Il prend l'esprit conforme à sa fin ", écrivait l'abbé Jean Dudouyt à $\mathrm{M}^{\mathrm{gr}}$ de Laval, en 1684; « il s'affectionne pour la mission du Canada et on ne peut pas faire davantage qu'a fait Monsieur l'abbé Brizacier pour nos affaires cette année. Il a dressé les lettres, les mémoires, il est venu à Versailles et s'intéresse et agit en tout ce qui regarde le Canada. Ainsi j'espère qu'on ne fera plus de distinction entre les missions de Chine, de la Perse et du Canada et qu'on embrassera tout également ${ }^{14}$." Le Séminaire de Paris venait précisément de décréter lui-même, cette année-là, l'envoi de trois de ses prêtres au $\mathrm{Ca}$ nada.

Cependant, le modus vivendi fixé par l'acte de désistement de 1682 ne vécut dans son intégrité que la courte période de dix années. On n'eut le temps d'élire le supérieur à Québec même qu'à trois reprises, en 1683 , 1685 et 1688. MM. Henri de Bernières et Louis Ango de Maizerets alternaient dans cette charge depuis la fondation et devaient le faire, du reste, jusqu'à 1721.

Des difficultés s'étant élevées entre le Séminaire de Québec et $M^{\text {gr }}$ de Saint-Vallier, peu de temps après l'accession de celui-ci au siège de Québec, difficultés portant en grande partie sur l'exercice de l'autorité épiscopale à l'égard du séminaire, on dut recourir à une commission d'arbitrage devant le roi. Dans le règlement signé des parties, le 13 janvier $1692^{15}$, où la deuxième demande de $\mathrm{M}^{\mathrm{gr}}$ de Saint-Vallier se lisait comme suit: « Que les supérieurs et directeurs du Séminaire soient réduits au nombre de cinq, nommés par les supérieurs des missions étrangères de Paris et approuvés par M. l'Évesque ", les commissaires d'arbitrage ont écrit en regard: "Accordé ». M. de Maizerets faisait par la suite ses commentaires sur ce point, disant ${ }^{16}:$ \& Il paraît par ce second article que son intention était de se rendre le maître absolu du Séminaire. *

13 Ibidem, cartable Séminaire 95, $\mathrm{n}^{\circ} 8$.

Ibidem, cartable Lettres N, no 77 (11 mars 1684), page 5 .

Ibidem, cartable Chapitre, $\mathrm{n}^{\circ} 142$.

Ibidem, cartable Séminaire 95, $\mathrm{n}^{\circ} 46$. 
Si tel était le cas, l'évêque remportait là une bien piètre victoire. Car, on pouvait s'y attendre, le Séminaire de Paris prendrait facilement parti pour le Séminaire de Québec, de préférence à l'évêque; et celui-ci, malgré son privilège de refuser le supérieur nommé, se trouvait tout de même, s'il voulait s'en prévaloir, obligé de poser en public un acte odieux et difficile à justifier. Le cas se réalisa dès l'année suivante, quand vint de Paris la nomination de l'abbé de Bernières, encore une fois, comme supérieur du Séminaire de Québec. Malgré l'animosité de l'évêque, qui, à ce moment même, fulminait l'interdit contre les trois plus anciens officiers du séminaire, il se vit bien forcé d'accorder son approbation, même s'il la limita à une année d'exercice ${ }^{17}$.

Pour ce qui nous concerne, à ce point de notre histoire, il reste que s'opérait, en 1692, un autre mouvement de bascule dans les relations entre le Séminaire de Québec et celui de Paris. Disons toutefois que ce devait être le dernier sous le régime français. Les officiers du Séminaire de Québec furent ensuite nommés invariablement par ceux des Missions Etrangères et il arriva même que le supérieur fut un nouveau venu, envoyé expressément de Paris.

Quand à l'administration matérielle et à la régie interne du Séminaire de Québec, les supérieurs de Paris ne s'en occupèrent que pour appuyer de leur influence et trouver des crédits, lorsque la situation financière devenait alarmante à Québec. On adjura bien souvent de réduire les dépenses excessives et les charités trop libérales. On conseilla ou on déconseilla de vendre tel ou tel fonds immobilier. C'était tout ce qu'on pouvait faire et parfois on n'était pas écouté, car les points de vue n'étaient pas les mêmes.

Comme l'union avec le Séminaire de Paris, confirmée par le roi, avait contribué à sauvegarder l'existence du Séminaire de Québec, dans le conflit avec $\mathbf{M}^{\text {gr }}$ de Saint-Vallier, elle y contribua pareillement, quand la chose se présenta, avec beaucoup moins d'acuité, toutefois, sous deux de ses successeurs : $\mathbf{M}^{\mathrm{gr}}$ Herman Dosquet et $\mathbf{M}^{\mathrm{gr}}$ Henri-Marie Dubreuil de Pontbriand.

Le premier, ayant été naguère agrégé au Séminaire des Missions Étrangères de Paris, affecta de se considérer comme un directeur du Séminaire de Québec, une fois installé évêque de cette ville. Mais il y perçut bientôt une certaine indépendance à son égard et fit des représentations sur l'union au Séminaire de Paris. Là-bas, on connaissait le personnage, qu'on traitait un peu comme un confrère, et on lui répondit negative sur les articles susceptibles d'affecter l'essence de l'union entre les deux séminaires; on ne lui céda que sur des détails, qui parurent ne

17 Archives de l'Archevêché de Québec, Registre A, page 560. 
point déroger au droit commun des séminaires et aux règlements du roi ${ }^{18}$.

Du temps de $\mathrm{M}^{\mathrm{gr}}$ de Pontbriand, en 1747 , le conflit débuta par un désaccord personnel avec le procureur, l'abbé André-Mathurin Jacrau. Celui-ci, au surplus, était curé d'office à la cathédrale, nommé par le séminaire, et devint supérieur intérimaire. Comme tel, il aurait, paraît-il, refusé à l'évêque le droit de regard sur la régie interne du séminaire et l'application des fondations. Sérieusement vexé et n'ayant pas obtenu satisfaction directement, $\mathrm{M}^{\mathrm{rg}}$ de Pontbriand rappliqua auprès des directeurs de Paris, avec l'aide d'un légiste, sur le fonds du problème, à savoir la dépendance du séminaire envers l'évêque, comme il croyait pouvoir l'exiger. On lui répondit poliment mais fermement, sur la base des usages établis. Après avoir préparé une réplique ${ }^{19}$, en 1749 , l'évêque abandonna la partie.

L'année suivante, en effet, c'était le Chapitre de la cathédrale qui déclarait la guerre au Séminaire de Québec, lui réclamant, entre autres choses, la possession de la cure de Québec et allant jusqu'à demander au roi de " déclarer nulle et abusive l'union du Séminaire de Québec à celui des Missions Étrangères établi à Paris ${ }^{20}$ „... Amené forcément à prendre parti entre les deux corps en conflit, $\mathrm{M}^{\mathrm{gr}}$ de Pontbriand, faisant litière de ses propres griefs antérieurs, se prononça finalement en faveur du Séminaire de Québec, dans une déclaration sans aucune équivoque $^{21}$. Cette nouvelle chicane du Lutrin, si l'on peut dire, un procès déjà profondément engagé en cour de France, demeura en suspens, sans dénouement, à cause des circonstances politiques: la recrudescence de la guerre franco-anglaise et la cession du Canada, en 1763.

Qu'allait-il advenir, sous les nouveaux maîtres du pays, de l'union entre les Séminaires de Québec et de Paris ? Chose surprenante, la correspondance ne subit aucune interruption, ni durant la guerre, ni durant les années cruciales qui suivirent, de 1759 à 1764 , celles du régime militaire d'occupation. Le gouverneur James Murray exerçait, il est vrai, une censure rigoureuse sur tout le courrier. En 1763, il parla même de l'interdire, mais sans aller jusqu'à l'application de cette mesure. L'attitude du Séminaire de Québec lui inspirait la sympathie, autant que l'ingérance de la France dans l'ordre des institutions pouvait lui répugner.

Le Séminaire de Paris dispensa d'abord à celui de Québec des condoléances et des conseils de prudence, ainsi celui de ne pas entreprendre 1734)

18 Archives du Séminaire de Québec, cartable Lettres M. $n^{\circ} 83$ (20 mai

i9 Ibidem, cartable Séminaire $5, \mathrm{n}^{\circ} 72 \mathrm{a}$.

20 Ibidem, cartable Chapitre, $\mathrm{n}^{\circ} 315$ (1756), page 19

21 Ibidem, $\mathbf{n}^{\circ} 190$, liasse A, page 18. 
de réparations considérables avant le traité de paix définitif. Ce qui n'empêcha cependant pas nos Messieurs de s'y lancer dans toute la mesure de leurs moyens.

Vint ensuite la nomination d'un supérieur, et c'est alors qu'on constata tout le précaire du nouvel ordre politique. M. Sébastien Pressart ayant terminé son terme réglementaire, les directeurs de Paris procédèrent à son remplacement, le 28 février 1762 . Mais, quand Murray apprit la chose, il fit venir chez lui les directeurs du Séminaire de Québec et déclara « qu'il ne souffrirait pas qu'on eût égard à la dite nomination non plus qu'à aucun ordre qui émanerait du Séminaire de Paris, jusqu'à l'arrangement à faire pour tout le Canada; qu'il ne s'opposait pas à ce que les directeurs du Séminaire fissent entre eux une élection, pourvu néanmoins qu'ils ne nommassent aucun des deux sujets nommés par le Séminaire de Paris (Pierre Maillard et, à son défaut, Henri-François Gravé), auxquels il donnait positivement l'exclusion ». Le 4 juillet 1762 , on élut donc, à Québec, l'abbé Urbain Boiret comme supérieur. Pour les mêmes raisons, il recevra un nouveau mandat de trois ans, le 24 juillet 1765 .

Quand il aura fait à son tour six ans de supériorat, se posera le même problème. Mais, enhardis sans doute par ces deux précédents, les directeurs du Séminaire de Québec se feront un nouveau règlement, approuvé par $\mathrm{M}^{\mathrm{gr}}$ Jean-Olivier Briand, et entérineront dans ce règlement une résolution de leur conseil du 19 août 1768 , où se lisent les lignes suivantes:

- Nous, ayant considéré qu'il nous est pour le présent impossible à cause des difficultés que nous fait sur cela le gouvernement Anglais de conserver avec le Séminaire des Missions Étrangères de Paris l'ancienne union et dépendance en vertu de laquelle nous devions recevoir de lui notre Supérieur, ainsi qu'il avait été réglé par Sa Majesté très chrétienne en $1692(\ldots)$, il a été conclu d'une voix unanime que demain, 20 août, à huit heures du matin, il serait procédé à l'élection d'un Supérieur et autres officiers pour le gouvernement du dit séminaire, suivant la forme prescrite dans le dit règlement. 》

Effectivement, ladite élection eut lieu, portant à la charge de supérieur M. Henri-François Gravé, l'un des deux candidats éliminés par Murray en 1762. Mais ce dernier n'était plus là et le nouveau gouverneur Carleton ne paraît pas s'être interposé. $M^{\mathrm{gr}}$ Briand approuva de sa main cette élection, au Grand Livre du conseil du séminaire.

Mais on n'avait pas agi de la sorte sans consulter de nouveau le supérieur du Séminaire de Paris. Celui-ci, l'abbé François Sorbier de Villars, avait répondu à l'abbé Gravé, le 4 mars $1768{ }^{22}$ :

22 Ibidem, cartable Lettres $S, n^{\circ} 54$. 
« Je suis incliné à croire que le Séminaire de Québec ayant été érigé en 1663 comme séminaire diocésain, il doit être aujourd'hui soumis à l'Évêque comme il l'était alors, vu que son union au Séminaire des Missions Érangères de Paris a cessé de droit et de fait. Le décret de l'érection dudit Séminaire de Québec en 1663 semble donc la loi unique qui doit vous diriger et vous régler tant pour le temporel que pour le spiri. tuel. »

Cette lettre prenait d'autant plus de signification, que M. de Villars, supérieur du Séminaire de Paris, avait vécu douze ans à Québec, de 1744 à 1756, dont six ans comme supérieur; il connaissait parfaitement la situation morale et juridique du séminaire. Rien d'étonnant qu'il ap. prouva toutes les procédures de ses anciens collègues, le printemps suivant, et n'eut que des mots de louange pour apprécier le résultat de l'élection et même la rédaction du nouveau règlement du Séminaire de Québec, qui consacrait l'émancipation et la séparation juridique de ce dernier d'avec le Séminaire de Paris ${ }^{23}$.

Mais les relations d'affaires et d'amitié n'ont pas cessé pour au. tant; pas plus que cette union spirituelle, datant de la fondation, en vertu de laquelle on acquittait réciproquement des messes pour chaque prêtre des deux séminaires venant à décéder. Cette union paraît s'être prolongée jusqu'à la Révolution française.

Pour le moment, c'était encore l'abbé de Villars qui, muni de procuration, s'occupait de percevoir les rentes du gouvernement royal et les revenus des prieurés dont jouissait le Séminaire de Québec en France; il rendait compte chaque année de sa gestion. Ces bénéfices, si modestes qu'ils fussent, étaient d'un prix inestimable à la période des grandes restaurations et de la réorganisation du Séminaire de Québec. On laissait les fonds déposés en France, où ils servaient à acquitter les factures de provisions, de tissus et de livres qu'il fallait importer chaque année. Le procureur du Séminaire, l'abbé André-Mathurin Jacrau, se rendit même en personne passer deux ans en France, de 1764, à 1766, tant pour refaire sa santé que pour s'occuper des affaires du Séminaire.

Après la mort de l'abbé de Villars, en 1788 , les relations se firent un peu moins fraternelles, mais demeurèrent sympathiques. D'ailleurs, les épreuves traversées par le Séminaire de Québec, lors de la Conquête, eurent bientôt leur pendant en France, lors de la Révolution. Tous les prêtres du Séminaire de Paris, sauf un vieillard impotent, crurent prudent de se disperser, puis de s'exiler en Angleterre. La sympathie des Messieurs de Québec les y accompagna, adoptant la forme concrète d'honoraires de messes, qu'on leur envoya à profusion, pour les aider dans leur dénûment, ainsi que d'autres compagnons d'exil.

23 Ibidem, Lettres $\mathrm{M}, \mathrm{n}^{\circ} 132$ (16 mars 1769). 
Parmi les prêtres français qui, d'Angleterre, se résolurent alors à passer au Canada, se trouvait un abbé Philippe-Jean-Louis Desjardins. Pour le Séminaire de Québec, son cas devint doublement intéressant. Avant de devenir l'agent qui fit parvenir au Canada quantité de précieux tableaux, rachetés à bon compte après la Révolution et où le Séminaire eut sa large part, l'abbé Desjardins fut aussi agrégé au séminaire, en 1800, et, forcé de repasser en France, en 1802, à cause de sa santé, il s'agrégea pareillement au Séminaire de Paris, rétabli par Napoléon, en 1805 , ce qui lui permit de prolonger les relations épistolaires entre les deux maisons jusqu'en 1823. Dans une lettre du 16 juin 1816, il écrivait: "Je porterai au tombeau le souvenir de tout ce que j'ai reçu de bonté, d'indulgence et de générosité au Canada, mais particulièrement au Séminaire de Québec, auquel je demande à rester toute ma vie agrégé . M. Chaumont (supérieur des Missions Étrangères) me charge bien de vous dire que l'union entre les deux maisons doit subsister et qu'entre vous et nous il n'y aura jamais de guerre nationale. *

Au moment où l'influence de l'abbé Desjardins allait s'éteindre, commençaient les tractations de l'abbé Jean-Baptiste Thavenet, un sulpicien, et de l'abbé Jean Holmes, de Québec, pour tenter de récupérer, avec l'appui de l'Angleterre, les sources de revenu perdues en France aux mains de la Révolution. On y trouva une occasion de garder contact avec le séminaire de la rue du Bac, jusqu'en 1844.

Il y out ensuite un point mort d'une trentaine d'années où l'on vécut un peu loin des yeux, loin du cœur, entre les deux maisons. L'on n'avait pas encore l'institution des cartes de Noël, pour forcer à revoir chaque année la liste des correspondants bien méritants... Les fêtes du deuxième centenaire des deux séminaires, en 1863 et 1864, se passèrent même sans qu'on pensât à une représentation de part et d'autre.

Ce furent plutôt les vicissitudes de l'histoire de France qui incitèrent un jour les Messieurs de Paris à renouer les contacts, en se reportant vers Québec, comme vers un refuge de paix et de prospérité. En effet, nous devions à notre éloignement et à notre changement d'allégeance, d'avoir échappé aux pires épreuves de la vieille mère patrie. Au milieu du siècle dernier, nous étions d'ores et déjà en avance sur nos cousins de France, dans les voies de la fortune, et considérés par eux avec raison comme de grands privilégiés.

En 1871, l'abbé Claude-Charles Dallet, vétéran des Missions Etrangères dans l'Hindoustan, entreprit donc un voyage dans les deux Amériques, cherchant des ressources pour réparer les pertes de son séminaire lors de la guerre franco-allemande et de la Commune de Paris, qui lui succéda, aux premiers mois de 1871. Les charités populaires pour les missions lointaines se trouvaient elles-mêmes lamentablement réduites, en France, par les malheurs des temps. 
L'abbé Dallet se présenta d'abord à Québec, logeant au séminaire. Puis, quand il eut fait, avec l'encouragement des évêques, une longue et fructueuse tournée, ses recettes furent laissées en dépôt à la procure de la maison. Le Séminaire lui-même y était allé du joli montant de $\$ 400$. De là débuta un échange intéressant et continu de visites, de politesses et de services mutuels, qu'il serait trop long de détailler. Les dépôts d'argent provisoires, étant devenus des dépôts à long terme, profitèrent aux deux institutions, le Séminaire de Paris y trouvant plus de sécurité et de rendement pour ses placements de fondations, le Séminaire de Québec s'y appuyant pour financer, entre autres, la construction du grand séminaire de 1880 . Et cela dura jusqu'à un dernier remboursement, le 24 juillet 1946 , le Séminaire de Paris ayant toutefois retiré le plus gros de ses crédits pendant la dévaluation du franc, au cours de la Première Grande Guerre.

Depuis le transfert en cour de Rome du procès de béatification de $\mathrm{M}^{\mathrm{gr}}$ de Laval, en 1883, c'était le procureur de la Société des Missions Étrangères résidant dans la Ville Éternelle qui s'acquittait avec zèle du rôle de postulateur de la cause.

Aux fêtes du troisième centenaire de la naissance de $\mathrm{M}^{\mathrm{gr}}$ de Laval, en 1923, les Missions Étrangères eurent comme représentant à Québec $\mathrm{M}^{\mathrm{gr}}$ Alexandre Berlioz et souscrivirent 500 francs pour les plaques commémoratives posées à Montigny-sur-Avre et à Saint-Germain des Prés. Dans son allocution, le représentant de Paris évoqua les anciennes relations entre les deux séminaires. Puis il ajoutait: " $\mathrm{Si}$, à ces souvenirs, je joins l'émotion que j'ai éprouvée en voyant, à l'entrée du Séminaire de Québec, le vieux chiffre de la Société des Missions Étrangères, S-M.E, vous devinez les sentiments inexprimables qui remplissent mon cœur. Dans ce chiffre de convention, il y a autre chose pour moi que des lettres juxtaposées ... C'est un pèlerinage que je suis venu faire au tombeau... du Vénérable François de Laval, membre de la Société des Missions Étrangères, Évêque de Pétrée, Vicaire Apostolique de la NouvelleFrance et premier Évêque de Québec .»

Malgré la solidité notoire des traditions et des institutions au Canada français, vous serez quand même étonnés d'apprendre que le séminaire que j'ai l'honneur de représenter ici fonctionne encore, devant la loi civile, sous la raison sociale de * Séminaire des Missions Étrangères de Québec " et sous l'égide des lettres patentes reçues de Louis XIV en avril 1663. Son existence civile a été respectée par la capitulation de Montréal et les instructions britanniques consécutives au traité de Paris; on a gardé cette charte telle quelle, malgré ses clauses désuètes, et on ne l'a amendée qu'accessoirement en 1843. Pourquoi changer ce qui était bien fait et solide? Y a-t-il d'autres institutions parmi nous qui puissent arborer un aussi vénérable privilège ? 
Cette appellation d'allure anachronique: Séminaire des Missions Étrangères de Québec, n'était pourtant pas une usurpation; elle n'était pas seulement l'écho fidèle des rêves apostoliques de $M^{\mathrm{gr}}$ de Laval, ni le fac-similé de quelques mots burinés dans les actes primitifs du séminaire. Elle correspondait à une réalité plus ou moins perdue de vue de nos jours, à un chapitre de notre histoire que personne d'autre n'évoquera, malheureusement, à ce congrès. Le Séminaire de Québec, pendant près d'un siècle, a bel et bien fait des missions. Collègue et partenaire du Séminaire de Paris, dans ce temps-là, il a envoyé et soutenu des missionnaires, soit en Acadie, soit au Mississipi. C'est seulement à cause des pertes essuyées à la Conquête et de la nouvelle fonction majeure qu'il a assumée dans l'œuvre d'éducation, qu'il n'a pu songer à reprendre cette vocation. Mais elle est restée gravée dans son blason, un acquit à son crédit dans l'histoire.

Et cette union du Séminaire de Québec avec celui de Paris, dont nous avons surtout épilogué devant vous, à quoi a-t-elle servi ? C'est la question que des gens sérieux, des théoriciens peuvent encore se poser. Il n'y a pas de balance pour les impondérables. Mais certaines valeurs spirituelles et morales, semées par la Providence sur le sentier des humains, ont un poids incalculable sur leur sort, qu'il s'agisse des individus ou des institutions.

L'union dont nous parlons, certes, a comporté des inconvénients. Il est bien sûr, entre autres, qu'il pouvait être agaçant, pour un évêque d'une autre mentalité que $\mathbf{M}^{\mathrm{gr}}$ de Laval, de trouver établi, dans son diocèse, un séminaire-communauté qui échappait partiellement à son contrôle, en relevant d'une autorité extérieure. Savoir alors faire taire son amour-propre et parvenir à gouverner quand même demandait à la fois de la vertu et de la diplomatie. La Grande Encyclopédie signale, au mot "Séminaires ", que des conflits semblables s'élevèrent en France, sous l'ancien régime, provoquant plus d'une fois les plaintes des assemblées du clergé.

Mais les avantages l'emportèrent sur les inconvénients, dont l'acuité, du reste, s'est fort atténuée avec le recul de l'histoire. Et c'est surtout le Séminaire de Québec qui fut bénéficiaire, à cause de l'éloignement et de la précarité de ses moyens. Il avait besoin, pourrions-nous dire, d'un appui solide extérieur, comme un jeune arbre a besoin d'un tuteur pour prendre racine et pousser son tronc droit et vigoureux. Il avait besoin d'appui matériel ; il avait besoin surtout de prêtres doctes et saints, de formateurs de prêtres, qu'il ne pouvait de longtemps trouver sur place, en pays neuf.

Le temps viendrait, précisément au bout d'un siècle, où la Providence, par la voix des événements et des personnages politiques, ferait tomber des liens devenus superflus, quoique toujours chers. Ce serait alors l'émancipation forcée. Mais, désormais plus solidaire avec l'évêque 
du lieu, le Séminaire de Québec pourrait déjà voler de ses propres ailes, ayant non seulement ses propriétés, son capital humain, mais son âme à lui et ses traditions établies.

Enfin, pour faire le joint avec la conférence qui va suivre et tout le congrès, disons que l'union des séminaires de Québec et de Paris aura fait sentir son influence bénéfique jusque dans la fondation de la Société des Missions Étrangères de la Province de Québec, il y a cinquante ans. Cette union, perpétuée, nous l'avons vu, par des relations moralement ininterrompues, aura été le fil d'Ariane conduisant sur nos plages des agents recruteurs des Missions Étrangères de Paris, comme l'abbé Paul Sibers, en 1918, et des fondateurs en perspective, comme $\mathrm{M}^{\mathrm{gr}} \mathrm{Jean}$ de Guébriant, en 1920. Leur unique succès, sinon leur faillite, sera d'alerter les évêques de la province de Québec et, par une réaction nationaliste bien légitime, de les inciter à faire plutôt une fondation canadienne.

"Ils ont pensé, écrira l'abbé Clovis Rondeau, en 1922, qu'un séminaire canadien dirigé par des prêtres canadiens, sous la tutelle de l'épiscopat de notre province pousserait des racines plus profondes dans notre sol et par conséquent rendrait plus de services aux missions 》 elles-mêmes ${ }^{24}$. L'expérience a magnifiquement confirmé leurs espérances et les Missions Etrangères de Paris ont eu le bon esprit de n'en jamais manifester aucun dépit.

Honorius Provost, ptre, archiviste du Séminaire de Québec.

24 Vers les terres d'infidélité (CEuvre des Tracts, $\mathrm{n}^{\circ}$ 39), Montréal, 1922, pages 5 et suiv. 